\title{
High-velocity Nal and Call absorption components observed towards the IC 443 SNR
}

\author{
B. Y. Welsh ${ }^{1}$ and S. Sallmen ${ }^{2}$ \\ ${ }^{1}$ Experimental Astrophysics Group, Space Sciences Laboratory, UC Berkeley, Berkeley, CA 94720, USA \\ 2 Department of Physics, Univ. of Wisconsin - La Crosse, La Crosse, WI 54601, USA
}

Received 7 May 2003 / Accepted 16 June 2003

\begin{abstract}
We present high-resolution spectra $\left(R \sim 1.8 \mathrm{~km} \mathrm{~s}^{-1}\right)$ of the interstellar NaI and CaII interstellar absorption lines observed towards 4 early-type stars with distances of 900-1500 pc in the line-of-sight towards the IC 443 Supernova Remant (SNR). The spectra of two of these stars (HD 43582 and HD 254577) exhibit a very complex pattern of absorption with cloud components covering a total velocity range of $-100 \mathrm{~km} \mathrm{~s}^{-1}$ to $+50 \mathrm{~km} \mathrm{~s}^{-1}$. The relative absorption strength of many of the higher velocity components is highly variable between these two stars, suggesting that the disturbed interstellar gas in this region possesses significant density gradients and/or large differences in ionization and element depletion. In addition, we have detected three additional high-velocity components at $V_{\text {helio }}=-97.5,-84.0$ and $-67.6 \mathrm{~km} \mathrm{~s}^{-1}$ solely in their CaII lines, suggesting that the very highest velocity gas is more ionized and/or warmer than the lower velocity components or it has a highly variable level of gas phase element abundances.

The column density ratios of $\mathrm{NaI} / \mathrm{CaII}$ for the higher velocity cloud components are all $<0.2$, which is consistent with appreciable levels of dust grain destruction due to interstellar shocks caused by interaction of the expanding SNR blast-wave with the ambient interstellar medium. The distance to IC 443 is confirmed at $\sim 1500 \mathrm{pc}$, which places the remnant at a similar distance to the Gem OB1 stellar association. Finally we note that the model of Chevalier (1999), in which the SNR is expanding into a clumpy interstellar medium, can best reproduce the observed patterns of both emission and absorption.
\end{abstract}

Key words. ISM: bubbles - ISM: supernova remnants

\section{Introduction}

IC $443\left(l=189.1^{\circ}, b=+3.0^{\circ}\right)$ is a relatively bright galactic supernova remnant (SNR) of age $\sim 30000$ yrs, with a diameter of $\sim 50$ arcmin and a distance estimate of $1.5 \mathrm{kpc}$ (Petre et al. 1988). It has a filamentary appearance in visible images, whereas infrared observations have revealed the presence of 3 shell-like interlocking cavities into which the supernova blast-wave appears to have expanded (Braun \& Strom 1986a). The interaction of the expanding SNR with the ambient interstellar medium (ISM) has been confirmed in two ways: (i) by the detection of shock-excited line emission from the $\mathrm{OH}, \mathrm{CO}$ and $\mathrm{H}_{2}$ lines which provide convincing evidence of a strong interaction with a surrounding molecular cloud (Cesarsky et al. 1999; van Dishoeck et al. 1993), and (ii) by both $21 \mathrm{~cm} \mathrm{HI}$ (Braun \& Strom 1986b) and H- $\alpha$ and NII (Meaburn et al. 1990) emission line studies of IC 443 which have revealed the presence of shocked and expanding tenuous gas with radial velocities in the range $V_{\text {helio }}=-200$ to $+250 \mathrm{~km} \mathrm{~s}^{-1}$. However, the true nature of this complex region of expanding shells has been revealed by ROSAT X-ray images that show that one of the shells is not part of the IC 443 SNR at all, but is due to a chance superposition with a separate, older SNR named G189.6+3.3

Send offprint requests to: B. Y. Welsh, e-mail: bwelsh@ssl. berkeley.edu
(Asaoka \& Aschenbach 1994). This latter SNR seems to be located just in front of the IC 443 SNR, with a molecular cloud of gas lying between both of these features.

Recent X-ray observations by the ASCA satellite have confirmed that the IC 443 complex is a "mixed morphology" SNR in which the dominant X-ray emission mechanism, which arises primarily from the swept-up interstellar material, is thermal in nature (Kawasaki et al. 2002). Maps of the X-ray softness-ratio reveal a shell-like structure that correlates well with the visible, $\mathrm{H}-\alpha$ emitting filaments, whereas the hard $\mathrm{X}$-ray emission is concentrated in the very center of the SNR. Thus, IC 443 appears to have a plasma structure that can be best described by a central hot ( 1 million $\mathrm{K})$ region that is surrounded by cooler, soft X-ray emitting shells that are interacting with the surrounding interstellar medium. The progenitor stars of both the IC 443 and G189.6+3.3 SNRs are probably members of the Gem OBI association, which would then provide a natural explanation for their spatial proximity. In order to test this theory we have selected early-type stars belonging to Gem OBI (Humphreys 1978) whose sightlines are near-coincident with the emission contours of the IC 443 SNR in order to sample their interstellar absorption characteristics using the visible NaI and CaII lines. Disturbed, high- and intermediate-velocity interstellar gas components surrounding SNRs have been previously detected in 
Table 1. Stellar target information.

\begin{tabular}{lccccc}
\hline \hline Star & $(l, b)$ & $m_{\mathrm{v}}$ & Sp & $E(B-V)$ & $\begin{array}{c}\text { distance } \\
(\mathrm{pc})\end{array}$ \\
\hline HD 42379 & $\left(189.3^{\circ},+1.34^{\circ}\right)$ & 7.43 & B1II & 0.59 & 1300 \\
HD 254755 & $\left(189.1^{\circ},+3.34^{\circ}\right)$ & 8.91 & O9V & 0.84 & 1450 \\
HD 254577 & $\left(189.3^{\circ},+3.09^{\circ}\right)$ & 9.19 & B0.5II & 1.05 & 1450 \\
HD 43582 & $\left(189.1^{\circ},+3.23^{\circ}\right)$ & 8.79 & BOIII & 0.67 & 1500 \\
\hline
\end{tabular}

absorption using these lines towards the Vela SNR (Wallerstein \& Silk 1971), the Monoceros Loop SNR (Wallerstein \& Jacobsen 1976), Shajn 147 (Silk \& Wallerstein 1973) and RCW 114 (Welsh et al. 2003). Although such visible observations can provide useful indicators concerning which sightlines contain disturbed neutral $($ I.P. $<11.8 \mathrm{eV})$ interstellar gas (presumably caused by the interaction between the expanding SNR shock and the ambient ISM), more detailed studies are best carried out at ultraviolet (UV) wavelengths which contain spectral lines that are sensitive to a wide range of ionization stages arising from a larger variety of chemical elements. When combined, the visible and UV absorption data can provide detailed information on the velocity structure, mass, energy, density, pressure and elemental abundance of the disturbed interstellar gas interacting with an expanding SNR (e.g. see Jenkins et al. 1984 for details on the Vela SNR).

This paper reports on the disturbed nature of the interstellar gas found towards the IC $443 \mathrm{SNR}$, as revealed by high resolution $\left(R \sim 1.8 \mathrm{~km} \mathrm{~s}^{-1}\right) \mathrm{NaI}$ and CaII absorption observations. Interstellar cloud components with velocities as high as $-100 \mathrm{~km} \mathrm{~s}^{-1}$ have been detected, and these observations will form the basis for a more extensive future study of these sightlines at ultraviolet wavelengths using the wide variety of spectral lines available to observations using both the NASA FUSE and HST Cosmic Origins Spectrograph instruments.

\section{Observations}

We have made observations towards four early-type stars, three of which have distances comparable to that of the IC 443 SNR, together with one star (HD 42379) that has been included as a probe of the foreground interstellar material. In Table 1 we list the relevant astronomical data for these stars, which includes their visual magnitudes, spectral types, reddening values and distance estimates. These values were taken from the on-line Simbad astronomical data-base, with the distance and reddening estimates (accurate to $\sim \pm 25 \%$ ) being taken from Odenwald \& Shivanandan (1985), Seab \& Snow (1984) and Rickard (1972). The positions of three of the targets with respect to the optical emission contours of the IC 443 SNR are shown in Fig. 1. The slightly foreground star HD 42379 lies approximately $2^{\circ}$ away from IC 443 and is thus not shown in this figure. Note that two of the target stars (HD 254577 and HD 43582) have sight-lines that are definitely contained within the SNR emission contours, whereas the HD 254755 sight-line appears to lie just beyond these limits.

Observations of both interstellar NaI D1 \& D2 lines at $\sim 5890 \AA$ A were obtained during the night of Dec. 30th, 2002 using the coude-echelle ("cs21") spectrograph (Tull et al. 1995)

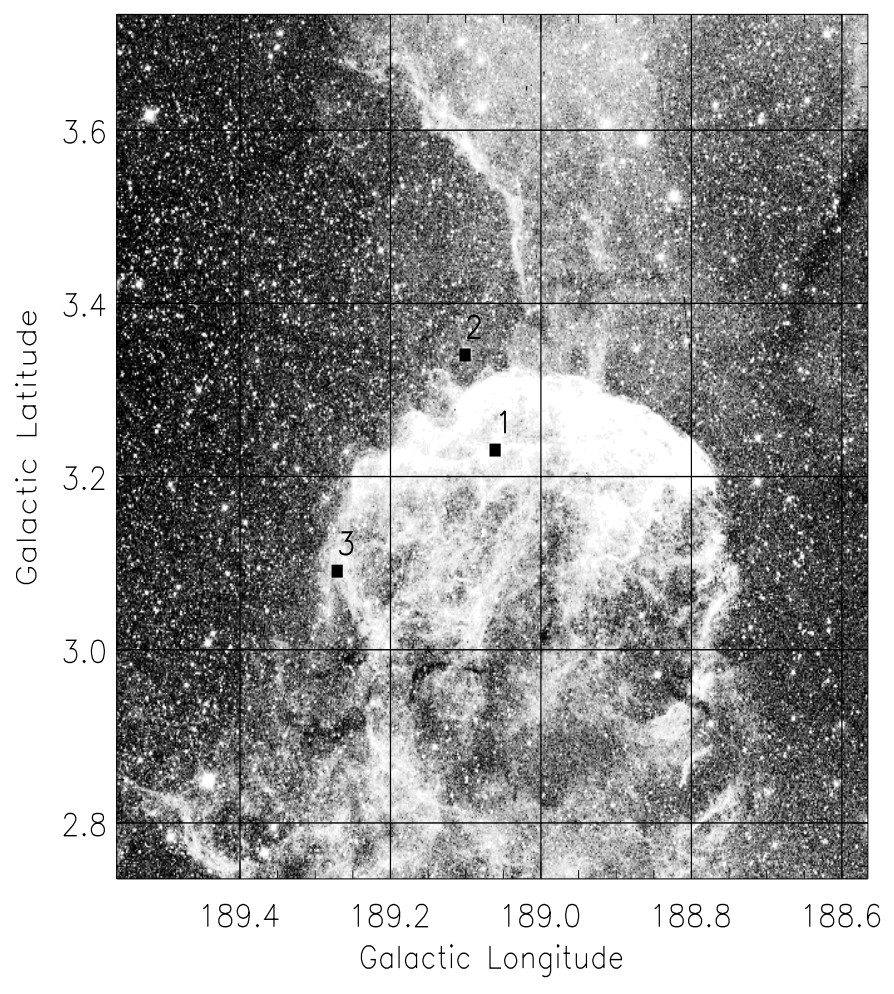

Fig. 1. Positions of the 3 stars (1) HD 43582, (2) HD 254755 and (3) HD 254577 with respect to the optical emission from the IC 443 SNR. The digitized Sky Survey image was taken from the SkyView web-site at http://skyview.gsfc.nasa.gov

at the $2.7 \mathrm{~m}$ telescope of the McDonald Observatory, Texas. The CaII K-line data were recorded on the nights of Jan. 1st $\& 2 \mathrm{nd}, 2003$ using the same instrumentation. The photon data were recorded with a Tektronix $2048 \times 2048$ CCD detector and the raw spectral images extracted using data reduction procedures analogous to those detailed in Sfeir et al. (1999). Briefly these software programs perform cosmic ray removal, background subtraction, flat-fielding, optimal spectral order extraction and wavelength assignment (from Th-Ar calibration spectra) on the recorded data. The resolution of the resultant spectra was $1.8 \mathrm{~km} \mathrm{~s}^{-1}$ and the wavelength accuracy of the calibrated data was $\sim \pm 0.02 \AA$. Typical total exposure times ranged from $1 \mathrm{~h}$ for the $\mathrm{NaI}$ data to $2 \mathrm{~h}$ for the CaII spectra. Due to the faintness of the targets and the high spectral resolution, the $\mathrm{S} / \mathrm{N}$ ratio of the recorded spectra were $\sim 15: 1$ for the NaI spectra and only 10:1 for the CaII data. However, this was deemed sufficient to isolate and detect any high-velocity absorption components in the spectra. All velocities quoted in this paper are reported in the heliocentric frame of reference. 

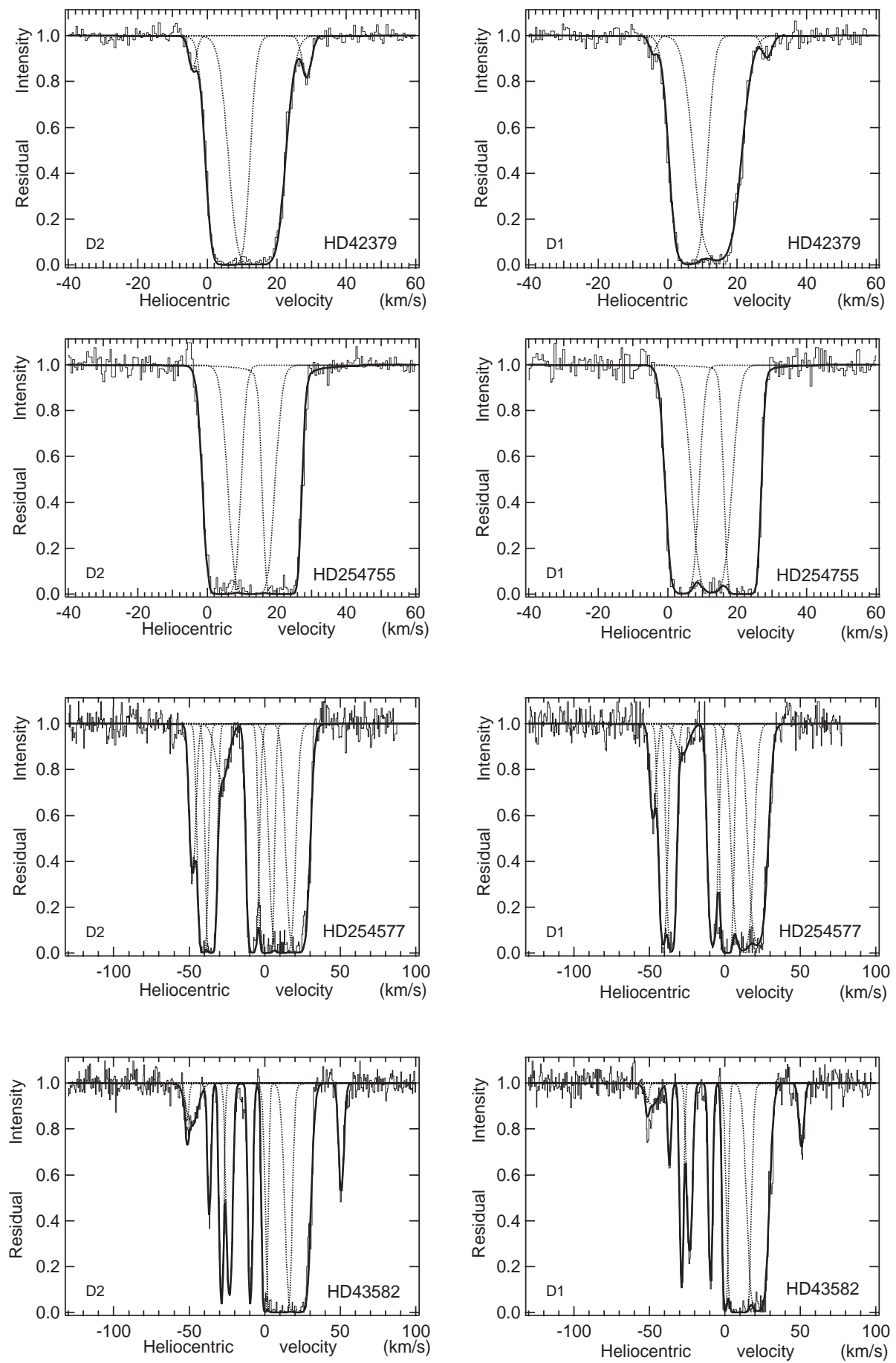

Fig. 2. Interstellar NaI D2 and D1 absorption lines observed towards the 4 target stars. Superposed on the residual intensity data points (light full line) is the multi-component best-fit absorption model (see Table 2). The dotted lines indicate each of the components used in this model.

Finally, we have removed the many narrow telluric water vapor absorption lines that particularly affect contamination of the NaI D line-profiles using a computed synthetic atmospheric transmission spectrum described in Lallement et al. (1993).

\section{Interstellar analysis}

We have determined the local stellar continua for all of the interstellar $\mathrm{NaI}$ and $\mathrm{CaII}$ absorption lines using a multi-order polynomial in order to produce the resultant residual intensity profiles shown in Figs. 2 and 3. These profiles were then fit with multiple absorption components (identified with interstellar gas "clouds") using a line-fitting program described in Sfeir et al. (1999). This program assigns a 3-parameter theoretical fit to the observed absorption profiles by assigning values for the interstellar gas cloud component velocity, $V$, a Gaussian velocity dispersion, $b$ and a cloud component column density, $N$. This fitting procedure works very well for absorption components that are not saturated, but the process leads to large uncertainties in the derived column densities for the central cores 

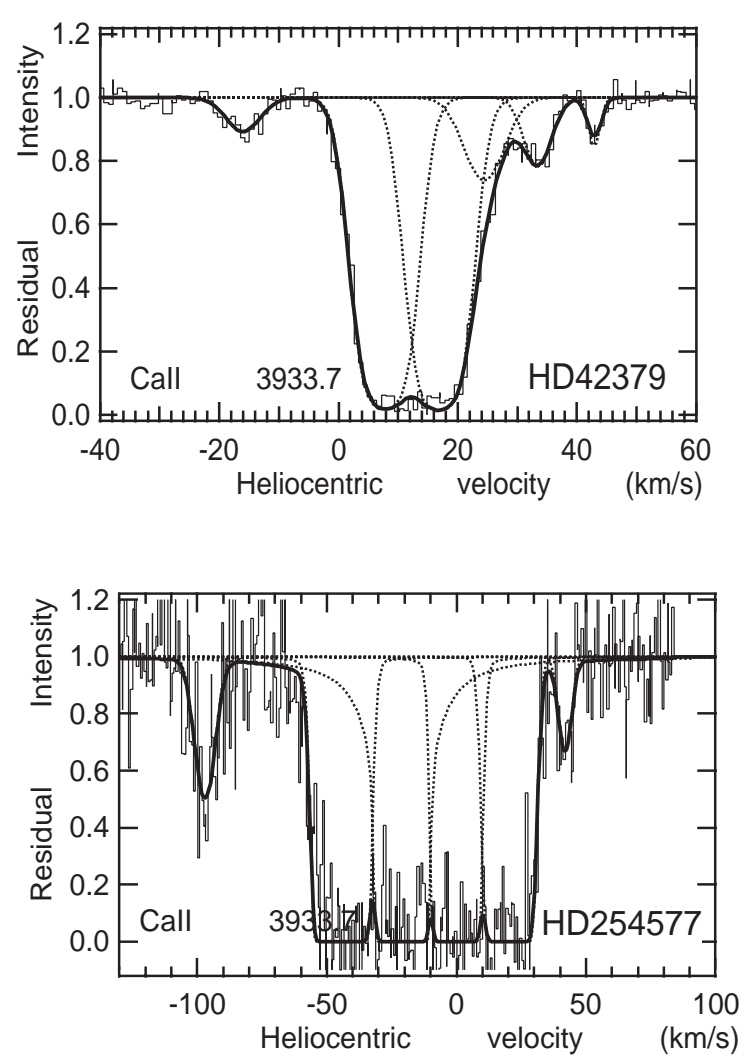
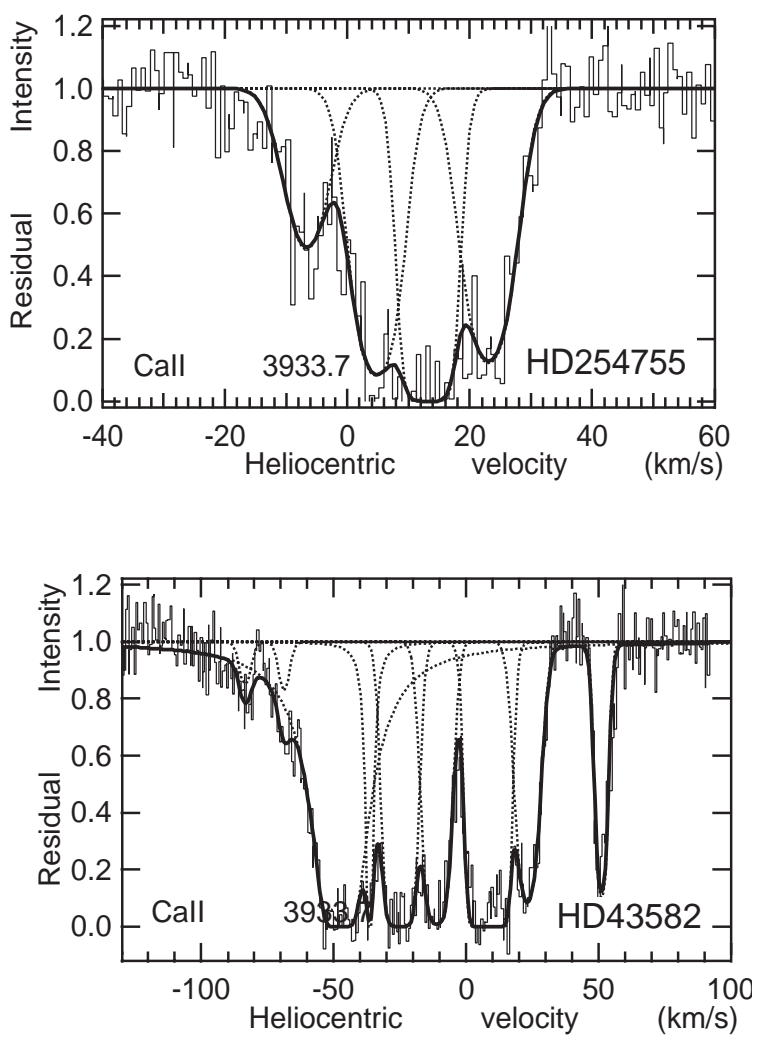

Fig. 3. Observed and model interstellar CaII-K absorption profiles for the 4 targets. Symbols are as stated in Fig. 2.

of spectral lines that possess very high values of saturation. In such cases (i.e. for the central line-core components) the bestfit values of $b$ and $N$ shown in Table 2 (and marked with *) are not well constrained and are listed only for the purpose of showing the velocity range over which the central absorption occurs. Fortunately, most of the absorption components located far from the saturated line-cores are unsaturated and reliable values of $V, b$ and $N$ are listed in Table 2 for both the NaI and CaII spectral lines. We note that although the absolute accuracy of the observed data wavelength scale is $\sim 0.02 \AA$ the accuracy of the velocities assigned to individual absorption components by the line-fitting software routine depends on several variables such as blending with nearby components and the local signalto-noise ratio of the data points. Thus, the model absorption component velocities $(V)$ listed in Table 2 are probably only accurate to $\pm 3.0 \mathrm{~km} \mathrm{~s}^{-1}$.

Our profile fits have been performed using the minimum number of absorption components. We have used the criterion of Vallerga et al. (1993) such that the addition of extra components (which will always improve the fit at some level) results in a reduction of the chi-squared residual error between the observed and computed data points of $>1 \%$. Errors for the derived component column densities (for unsaturated components) are also listed in Table 2. All of these model fits are shown superposed on the observed profiles in Figs. 2 and 3.

\section{Discussion}

It is immediately apparent from Figs. 2 and 3 that the $\mathrm{NaI}$ and CaII absorption profiles recorded towards both HD 254577 and
HD 43582 are far more complex and span a much wider range in velocity than the profiles recorded towards the other two targets. We note that the star HD 42379 lies $\sim 150 \mathrm{pc}$ foreground to the SNR and thus it is not unexpected that its observed absorption profiles are less complex than the more distant targets. Also, although HD 254755 lies at a distance that is comparable to IC 443, its sight-line does not pass through the emission contours of the SNR (see Fig. 1) and thus, again we might expect to observe fewer absorption components.

All of the "extra" absorption components observed towards the two stars HD 254577 and HD 43582 are formed at velocities either more negative than $V=-30 \mathrm{~km} \mathrm{~s}^{-1}$ or more positive than $V=+45 \mathrm{~km} \mathrm{~s}^{-1}$. Such high values of absorption component velocity are greater than those normally found in the general ISM and are inconsistent with absorption due to circumstellar gas or the expansion of a stellar HII region. Thus, since both stars have sight-lines that apparently intersect with the emission contours of IC 443 and both are at a distance $\sim 1500 \mathrm{pc}$, we can confidently conclude that these higher velocity absorption components are most probably caused by the interaction of the expanding SNR with the surrounding ISM.

\subsection{The Nal D-line absorption spectra}

In Fig. 2 we see that all 4 stars show strong central absorption over the velocity range of -5 to $+30 \mathrm{~km} \mathrm{~s}^{-1}$, which we associate with absorption due to the foreground line-of-sight interstellar medium towards these targets. The absorption components formed over this velocity range are all saturated and will not be discussed further in this paper, apart from our assumption that 
Table 2. NaI and CaII absorption line best-fit parameters (stars listed by increasing distance).

\begin{tabular}{|c|c|c|c|c|c|c|c|c|}
\hline Star & $\begin{array}{c}V \\
\mathrm{~km} \mathrm{~s}^{-1}\end{array}$ & $\bar{b}$ & $\begin{array}{c}N \\
\left(10^{10} \mathrm{~cm}^{-2}\right)\end{array}$ & & $\begin{array}{c}V \\
\mathrm{~km} \mathrm{~s}^{-1}\end{array}$ & $\bar{b}$ & $\begin{array}{c}N \\
\left(10^{10} \mathrm{~cm}^{-2}\right)\end{array}$ & $\mathrm{NaI} / \mathrm{CaII}$ \\
\hline \multicolumn{9}{|l|}{ HD 42379} \\
\hline \multirow[t]{6}{*}{...NaI... } & -4.2 & 1.5 & $5.1 \pm 1.4$ & ...CaII... & -15.8 & 3.9 & $11.8 \pm 2.0$ & - \\
\hline & +5.5 & $3.7 *$ & $>600^{*}$ & & +8.0 & 4.6 & $505 \pm 40$ & $>1.2$ \\
\hline & +13.9 & $5.7 *$ & $>800^{*}$ & & +17.2 & 4.6 & $500 \pm 50$ & $>1.6$ \\
\hline & & & & & +24.8 & 4.5 & $35 \pm 8$ & - \\
\hline & +28.9 & 1.8 & $7.6 \pm 1.8$ & & +33.7 & 3.0 & $20 \pm 4.5$ & 0.38 \\
\hline & & & & & +43.1 & 1.3 & $5.8 \pm 1.0$ & - \\
\hline \multicolumn{9}{|l|}{ HD 254755} \\
\hline \multirow[t]{4}{*}{...NaI... } & & & & ...CaII... & -6.5 & 4.6 & $88 \pm 5$ & - \\
\hline & +4.6 & $3.1^{*}$ & $>850 *$ & & +5.0 & 4.1 & $275 \pm 50$ & $>3.1$ \\
\hline & +13.1 & $4.0 *$ & $>750^{*}$ & & +13.5 & $3.0 *$ & $>1500^{*}$ & - \\
\hline & +21.9 & $2.4^{*}$ & $>2000 *$ & & +23.0 & 4.5 & $255 \pm 20$ & $>7.8$ \\
\hline \multicolumn{9}{|l|}{ HD 254577} \\
\hline \multirow[t]{10}{*}{...NaI... } & & & & ...CaII... & -97.0 & 5.0 & $90 \pm 15$ & - \\
\hline & -48.1 & 2.6 & $50 \pm 10$ & & & & & \\
\hline & -41.1 & 2.6 & $320 \pm 50$ & & -44.5 & $4.5^{*}$ & $>4000^{*}$ & $<0.08$ \\
\hline & -35.7 & 2.6 & $505 \pm 200$ & & & & & \\
\hline & -28.2 & 5.7 & $29.5 \pm 7$ & & -21.0 & $3.5 *$ & $>5000^{*}$ & - \\
\hline & -8.2 & 3.1 & $380 \pm 100$ & & & & & \\
\hline & +1.1 & $2.9^{*}$ & $>1500^{*}$ & & 0.0 & $4.0 *$ & $>4500 *$ & - \\
\hline & +11.5 & $6.0^{*}$ & $>900 *$ & & & & & - \\
\hline & +21.7 & $5.7 *$ & $>600^{*}$ & & +20.5 & $4.8^{*}$ & $>5500^{*}$ & - \\
\hline & & & & & +42.0 & 3.2 & $25.0 \pm 5$ & - \\
\hline \multicolumn{9}{|l|}{ HD 43582} \\
\hline \multirow[t]{12}{*}{...NaI... } & & & & ...CaII... & -84.0 & 2.5 & $12 \pm 2$ & - \\
\hline & & & & & -67.6 & 4.5 & $40 \pm 6$ & - \\
\hline & -54.0 & 0.3 & $0.35 \pm 0.1$ & & -58.1 & 3.3 & $60 \pm 12$ & 0.006 \\
\hline & -48.0 & 4.5 & $20.5 \pm 4$ & & -47.2 & $2.4^{*}$ & $>5000^{*}$ & $<0.004$ \\
\hline & -37.2 & 1.1 & $24.0 \pm 5$ & & - & & & \\
\hline & -29.1 & 1.1 & $146 \pm 31$ & & -28.1 & $4.6^{*}$ & $>2000 *$ & $<0.07$ \\
\hline & -23.9 & 2.0 & $105 \pm 21$ & & & & & - \\
\hline & -10.0 & 1.2 & $136 \pm 30$ & & -10.9 & 4.5 & $650 \pm 200$ & 0.21 \\
\hline & +1.4 & $1.7^{*}$ & $>1000^{*}$ & & & & & - \\
\hline & +11.1 & $4.9^{*}$ & $>1000^{*}$ & & +8.6 & $4.5^{*}$ & $>5000^{*}$ & - \\
\hline & +22.6 & $5.0^{*}$ & $>650^{*}$ & & +23.4 & 4.6 & $310 \pm 50$ & $>2.0$ \\
\hline & +50.2 & 2.1 & $27.5 \pm 6$ & & +51.5 & 3.2 & $145 \pm 20$ & 0.19 \\
\hline
\end{tabular}

* Saturated component.

the center of this absorption is located at $V_{\text {helio }} \sim+10 \mathrm{~km} \mathrm{~s}^{-1}$. The sight-line towards the nearest star, HD 42379, contains a well-resolved component at $V=+28.7 \mathrm{~km} \mathrm{~s}^{-1}$. This sightline, which lies $\sim 2.5^{\circ}$ away from IC 443 , passes through the Gem OB1 association which contains several groups of earlytype stars with distances ranging from 1 to $2 \mathrm{kpc}$ (Carpenter et al. 1995). Thus, it seems likely that this velocity component is caused by expanding gas due to the combined stellar windpower of the OB-type stars.

The interstellar NaI spectrum of HD 254755 has none of the additional components seen towards HD 254577 and HD 43582. Its absorption spectrum is qualitatively similar to that of the foreground star, HD 42379, and thus is of little relevance to our discussion of the SNR. The sight-lines to the two most distant stars reveal several additional $\mathrm{NaI}$ components at velocities of $V \sim-55,-49$, and $+50 \mathrm{~km} \mathrm{~s}^{-1}$ which are also detected at similar velocities in their CaII spectra. This pattern of absorption is consistent with the picture of IC 443 described in the Introduction, in which the SN blast-wave has expanded into several interlocking shell-like cavities. These expanding shells, as revealed by the NaI observations, are composed mainly of neutral interstellar gas, supporting the view of both Cesarsky et al. (1999) and van Dishoeck et al. (1993) that the expanding SNR has collided with surrounding molecular clouds.

Although the NaI spectra of HD 43582 and HD 254577 are similar in many respects, the relative strengths of their higher velocity components are quite different. For instance, the $V=$ $+50 \mathrm{~km} \mathrm{~s}^{-1}$ component is only detected towards HD 43582 , which could be interpreted as being due to its greater distance behind the SNR. However, the absorption components formed at negative velocities towards HD 43582 are all weaker than the equivalent components detected towards HD 254577. This difference is absorption strength may be indicative of the presence of large density gradients throughout the SNR. Such gradients could be physically real, i.e. there are large density fluctuations in the gas clouds surrounding the SNR, or they could also be 
due viewing effects since the sight-line to HD 254577 (which is at the edge of the SNR) samples a greater depth through the SNR shell. We note that a similar pattern of interstellar inhomogeneity has been observed towards the Vela SNR by Jenkins et al. (1976). Alternately, the variations in the strength of the higher velocity components seen towards HD 43582 and HD 254577 could also be produced by depletion and/or ionization effects of the disturbed gas. Further observations using ultraviolet absorption lines would provide valuable information on this point.

\subsection{The Call K-line absorption spectra}

Figure 3 shows the strong central core (foreground line-ofsight) absorption for all 4 targets being formed over a similar velocity range to that of the $\mathrm{NaI}$ spectra (i.e. -5 to $+30 \mathrm{~km} \mathrm{~s}^{-1}$ ). The CaII spectrum of the foreground star, HD 42379, shows absorption components away from the central core at $V=-16$, +33 and $+43 \mathrm{~km} \mathrm{~s}^{-1}$, only one of which (at $V=+28.7 \mathrm{~km} \mathrm{~s}^{-1}$ ) is seen in the NaI spectra. Thus, these additional components may originate in a more ionized medium than that revealed by the NaI lines, perhaps gas ionized by the Gem OB1 early-type stars.

We find, again, that the star HD 254755 has none of the additional absorption components observed towards HD 254577 and HD 43582, confirming that its sight-line does not pass through the expanding nebular gas associated with IC 443.

Although the CaII spectrum of HD 254577 is of low $\mathrm{S} / \mathrm{N}$ ratio, a high-velocity component at $V=-97.5 \mathrm{~km} \mathrm{~s}^{-1}$ is prominent, as is a component at $V=+42 \mathrm{~km} \mathrm{~s}^{-1}$. Interestingly, neither of these absorption components is detected in the corresponding $\mathrm{NaI}$ spectra, suggesting that either (i) both components consist of ionized (and/or warmer) interstellar gas, or (ii) that both components posses highly variable levels of gas-phase abundances of $\mathrm{Ca}$ and $\mathrm{Na}$. The CaII spectrum of HD 43582 also contains several absorption components with unusually high velocities $(V=-84,-67,-58$ and $+51.5 \mathrm{~km} \mathrm{~s}^{-1}$ ). Of these components only the $V=-58$ and $+51.5 \mathrm{~km} \mathrm{~s}^{-1}$ clouds are detected at similar velocities in the $\mathrm{NaI}$ spectra, suggesting they are formed in a more neutral medium than the velocity components with the highest measured velocities.

\subsection{The Nal/Call ratios}

The column density ratio of $N(\mathrm{NaI}) / N(\mathrm{CaII})$ has long been used as a diagnostic of interstellar gas cloud components (Routly \& Spitzer 1952). Values of this ratio have been found to span several orders of magnitude (from 0.01 to $>100$ ), with the lowest values being associated with interstellar clouds of higher velocities (Siluk \& Silk 1974). The variation in the column density ratio is thought to be linked to the highly variable amount of depletion of interstellar $\mathrm{Ca}$ into dust grains. High-velocity clouds can produce interstellar shocks that are responsible for the sputtering of interstellar dust grains, which returns the $\mathrm{Ca}$ into the gas phase, thus producing a low value in the observed $\mathrm{NaI} / \mathrm{CaII}$ ratio.
Reliable values of the NaI/CaII column density ratio for the 4 targets are only available for absorption components that are well resolved and detected in both the $\mathrm{NaI}$ and $\mathrm{CaII}$ lines at similar component velocities. Unfortunately, due to the extent of saturation of the observed absorption lines, these criteria are met in only a few cases (see Table 2 ).

In the 4 cases for absorption components with similar $\left( \pm 5 \mathrm{~km} \mathrm{~s}^{-1}\right)$ cloud component velocities that lie beyond the central range of -40 to $+40 \mathrm{~km} \mathrm{~s}^{-1}$ (seen towards HD 254577 and HD 43852), we find NaI/CaII ratios $<0.2$. This low ratio would seem to confirm the presence of interstellar shocks throughout this disturbed region, as previously observed in both molecular $\mathrm{CO}$ and $\mathrm{H}_{2}$ emission studies (van Dishoeck et al. 1993). However, we note that in two of these four cases the velocity of the $\mathrm{NaI}$ and CaII components differs by $4 \mathrm{~km} \mathrm{~s}^{-1}$ and thus it is possible that the two kinds of absorption being compared may not actually arise in the same physical location.

\subsection{Age, dynamics and distance}

The dynamics and evolution of a typical SN explosion expanding into its ambient interstellar medium has been investigated theoretically by many authors (Chevalier 1974; Cioffi et al. 1988; Truelove \& McKee 1999). In the most simple case of expansion into a uniform medium of ambient density $1 \mathrm{~cm}^{-3}$, Chevalier (1974) gives the SNR shell velocity, $V$, as a function of the remnant age, $t$ (in units of $10^{5} \mathrm{yrs}$ ) as:

$V=66.5 \times t^{-0.69} \mathrm{~km} \mathrm{~s}^{-1}$.

Therefore, for an expansion velocity of $110 \mathrm{~km} \mathrm{~s}^{-1}$ with respect to the central rest velocity of the remnant, we derive an age of $t=4.8 \times 10^{4} \mathrm{yrs}$. This is in broad agreement with age estimates of $\sim 30000$ yrs based on X-ray observations by Petre et al. (1988), Asaoka \& Aschenbach (1994).

However, the observed morphology of IC 443 suggests that it cannot be modelled as a simple case of a SNR. Our absorption data reveal at least three major shell components expanding at $V \sim-95,-60$ and $+50 \mathrm{~km} \mathrm{~s}^{-1}$, which are detected towards both HD 254577 and HD 43582 (the two most distant targets observed). Expanding gas shells with similar velocities have also been observed in emission towards IC 443 in the HI survey of the SNR by Braun \& Strom (1986a) and in H- $\alpha$ echelle spectroscopy by Meaburn et al. (1990). Also, recent observations of the far IR emission lines of OI and CII towards IC 443 by Haas et al. (2003) have revealed blue-shifted profiles at a velocity of $-40 \mathrm{~km} \mathrm{~s}^{-1}$, consistent with the intermediatevelocity absorption components observed by us towards these two stellar targets.

Chevalier (1999) has recently modeled the evolution of a typical SNR expanding into a clumpy interstellar medium composed of molecular clouds; a situation physically similar to that of IC 443. In this model the interaction of the radiative shell(s) with surrounding molecular clumps of gas can produce shock fronts that are driven by considerable overpressure compared to the rest of the remnant. Such a model, in which a $100 \mathrm{~km} \mathrm{~s}^{-1}$ shell is expanding into a region of ambient density $\sim 15 \mathrm{~cm}^{-3}$, can adequately explain the levels of molecular emission observed from IC 443 by both Richter et al. (1995) 
and Moorhouse et al. (1991). This radiative shell model also implies an age of $30000 \mathrm{yrs}$ for the IC 443 remnant, under the assumption of a shell of radius $7.4 \mathrm{pc}$ expanding with a velocity of $100 \mathrm{~km} \mathrm{~s}^{-1}$ (as presently observed by us in the CaII line detected towards HD 254577).

Finally, we note that the distance to IC 443 is confirmed at $\sim 1.5 \mathrm{kpc}$. Reliable distances to SNRs are always difficult to obtain from either radio or X-ray measurements. The complex nature of the absorption profiles presently observed towards both HD 43582 and HD 254577, whose distances are 1450-1500 pc, clearly confirm the long-held belief that the IC $443 \mathrm{SNR}$ is at the same distance as the Gem OB1 stellar association.

\section{Conclusion}

We have obtained high-resolution $\left(1.8 \mathrm{~km} \mathrm{~s}^{-1}\right)$ spectra of the interstellar $\mathrm{NaI}$ and $\mathrm{CaII}$ absorption lines seen towards 4 stars with distances raging from $900-1550 \mathrm{pc}$ in the general sightline towards the IC 443 SNR. The greatest complexity of absorption profiles, in which components are observed over a velocity range of -100 to $+50 \mathrm{~km} \mathrm{~s}^{-1}$, has been detected towards the two most distant targets, HD 43582 and HD 254577. The sight-lines towards both stars are coincident with $\mathrm{H}-\alpha$ and HI emission from the SNR, which strongly supports the hypothesis that the absorption we have detected towards these two stars originates in disturbed gas associated with the expansion of the IC 443 SNR.

We have fit the absorption components observed in the $\mathrm{NaI}$ and CaII lines with models of cloud-component velocity, doppler broadening and column density. Although the NaI spectral profiles of HD 43582 and HD 254577 are similar, the relative strengths of their higher velocity components are quite different, suggesting that appreciable gas density gradients may exist throughout the SNR. Alternately, this effect could be caused by differences in element depletion and/or ionization of the disturbed gas. The highest velocity components, at $V_{\text {helio }}=-97.5,-84$ and $-68 \mathrm{~km} \mathrm{~s}^{-1}$, have only been detected in the interstellar CaII lines, suggesting that these cloud components are either more ionized and/or warmer than the components detected solely by the NaI lines, or they are formed in regions of highly variable gas phase element abundances.

The column density ratios of $N(\mathrm{NaI}) / N(\mathrm{CaII})$ for the higher velocity components are $<0.2$, implying that appreciable levels of dust grain destruction due to interstellar shocks from the SNR blast wave may be present. We note that the model of Chevalier (1999), in which the SNR is expanding into a clumpy ambient interstellar medium can best reproduce the observed patterns of both emission and absorption seen towards IC 443 . In such a model the SNR has an age of $\sim 30000 \mathrm{yrs}$, has a shell radius of $7.4 \mathrm{pc}$ and is expanding at a velocity of $\sim 100 \mathrm{~km} \mathrm{~s}^{-1}$ into a region of ambient density $\sim 15 \mathrm{~cm}^{-3}$. It should be stressed that the presently observed complexity of absorption components seen towards IC 443 merely emphasizes the extent of the complicated morphology of this highly disturbed region, in which the combined action of $\mathrm{SNe}$ and stellar winds haveproduced the numerous expanding interstellar shells and cavities.

Acknowledgements. We are grateful to the staff and directorate of the University of Texas McDonald Observatory. In particular we wish to thank both Prof. D. Lambert (Texas) and Prof. S. Federman (Toledo) for their patience in training us as users of the excellent spectroscopic instrumentation at the Observatory. We also thank L. Hobbs, whose useful comments considerably improved the final version of this paper. BYW and SS acknowledge funding from the NASA FUSE project under contract NAS5-32985 to the Johns Hopkins University.

\section{References}

Asaoka, I., \& Aschenbach, B. 1994, A\&A, 284, 573

Braun, R., \& Strom, R. 1986, A\&A, 164, 193

Braun, R. \& Strom, R. 1986, A\&AS, 63, 345

Carpenter, J., Snell, R., \& Schloerb, F. 1995, ApJ, 445, 246

Cesarsky, D., Cox, P., Pineau de Forets, G., et al. 1999, A\&A, 348, 945

Chevalier, R. 1974, ApJ, 188, 501

Chevalier, R. 1999, ApJ, 511, 798

Cioffi, D., McKee, C., \& Bertschinger, E. 1988, ApJ, 334, 252

Crawford, I., Lallement, R., Price, R., et al. 2002, MNRAS, 337, 720

Haas, M. R., Higdon, S., Burton, M, \& Hollenbach, D. 2003, BAAS, 30.08

Humphreys, R. 1978, ApJS, 38, 309

Jenkins, E., Silk, J., \& Wallerstein, G. 1976, ApJS, 32, 681

Jenkins, E., Wallerstein, G., \& Silk, J. 1984, ApJ, 278, 649

Kawasaki, M., Ozaki, M., Nagase, F., et al. 2002, ApJ, 572, 897

Lallement. R., Bertin, P., Chassefiere, E., \& Scott, N. 1993, A\&A, 271, 734

Meaburn, J., Whitehead, M., Raymond, J., Clayton, C., \& Marston, A. 1990, A\&A, 227, 191

Moorhouse, A., Brand, P., Geballe, T., \& Burton, M. 1991, MNRAS, 253, 662

Odenwald, S., \& Shivanandan, K. 1985, ApJ, 292, 460

Petre, R., Szymkowiak, A., Seward, F., \& Willingdale, R. 1988, ApJ, 335,215

Richter, M., Graham, J., \& Wright, G. 1995, ApJ, 454, 277

Rickard, J. 1972, A\&A, 17, 425

Routly, P., \& Spitzer, L. 1952, ApJ, 115, 227

Seab, C., \& Snow, T. 1984, ApJ, 277, 200

Sfeir, D., Lallement, R., Crifo, F., \& Welsh, B. Y. 1999, A\&A, 346, 785

Silk, J., \& Wallerstein, G. 1973, ApJ, 181, 799

Siluk, R., \& Silk, J. 1974, ApJ, 192, 51

Truelove, J., \& McKee, C., ApJS, 120, 299

Tull, R., MacQueen, P., Sneden, C., \& Lambert, D. 1995, PASP, 107, 251

Vallerga, J.V., Vedder, P., Craig, N., \& Welsh, B. Y. 1993, ApJ, 411, 729

van Dishoeck, E., Jansen, D., \& Phillips, T. 1993, A\&A, 279, 541

Wallerstein, G., \& Jacobsen, T. 1976, ApJ, 207, 53

Wallerstein, G., \& Silk, J. 1971, ApJ, 170, 289

Welsh, B. Y., Sallmen, S., Sfeir, D., \& Lallement, R. 2002, A\&A, 391, 705

Welsh, B. Y., Sallmen, S., Jelinsky, S., \& Lallement, R. 2003, A\&A, in press 